\title{
Charge Formation, Recombination, and Sweep-Out Dynamics in Organic Solar Cells
}

\author{
Sarah R. Cowan, Natalie Banerji, Wei Lin Leong, and Alan J. Heeger*
}

This manuscript is dedicated to the memory of Professor Alan G. MacDiarmid

This article presents a critical discussion of the various physical processes occurring in organic bulk heterojunction (BHJ) solar cells based on recent experimental results. The investigations span from photoexcitation to charge separation, recombination, and sweep-out to the electrodes. Exciton forma-

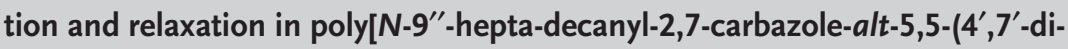
2-thienyl-2', $1^{\prime}, 3^{\prime}$-benzothiadiazole) (PCDTBT) and poly-3 (hexylthiophene) (P3HT) are discussed based on a fluorescence up-conversion study. The commonly accepted paradigm describing the conversion of incident photons into charge carriers in the $\mathrm{BHJ}$ material is re-examined in light of these femtosecond time-resolved measurements. Transient photoconductivity, timedelayed collection field, and time-delayed dual pulse experiments carried out on $\mathrm{BHJ}$ solar cells demonstrate the competition between carrier sweep-out by the internal field and the loss of photogenerated carriers by recombination. Finally, an emerging hypothesis is discussed: that bimolecular recombination accounts for the majority of recombination from short circuit to open circuit in optimized solar cells, and that bimolecular recombination is biasand charge-density-dependent. The study of recombination loss processes in organic solar cells leads to insights into what must be accomplished to achieve the "ideal" solar cell. gaps of the semiconducting polymers. Moreover, these new semiconducting polymers stabilize the photoinduced charge transfer. ${ }^{[5]}$ Finally, work on the stabilization of electrode ${ }^{[6-8]}$ and encapsulation ${ }^{[9]}$ materials has resulted in reports of extended lifetimes on the order of several years. The ultimate lifetime of organic solar cells is not yet known.

Within the field of photovoltaics, organic bulk heterojunction (BHJ) solar cells remain of interest because of the inexpensive processing techniques ${ }^{[10]}$ capable of producing large area modules-inkjet printing, ${ }^{[11]}$ screen printing, ${ }^{[12,13]}$ spray coating, ${ }^{[14,15]}$ roll-to-roll printing, ${ }^{[16]}$ flexographic printing, and even paint brush application. ${ }^{[17]}$ Laboratory research has primarily focused on reproducible and small-area casting techniques such as spin-coating, ${ }^{[18]}$ drop-casting, and blade-coating. ${ }^{[19]}$ In addition, the chemical elements which compose these devices are readily available in abundance in the earth's surface, making organic photovoltaics a scalable technology. In the current energy climate, there exist opportunities for a diverse number of energy

\section{Introduction}

Polymer solar cells celebrate a sixteenth birthday this year. The field has achieved several critical milestones in the past couple of years. Internal quantum efficiency of almost unity has been demonstrated. ${ }^{[1]}$ National Renewable Energy Lab (N.R.E.L.)certified power conversion efficiencies surpassing $8 \%$ have been achieved by two groups with device area in excess of $1 \mathrm{~cm}^{2}{ }^{2}{ }^{[2,3]}$ The highest reported power conversion efficiency in peer-reviewed literature is $7.4 \% .{ }^{[4]}$ High efficiency devices have been made possible by advances in the chemical synthesis of donor-acceptor copolymers with improved absorption coefficient and reduced energy

Dr. S. R. Cowan, Dr. N. Banerji, Dr. W. L. Leong, Prof. A. J. Heeger Center for Polymers and Organic Solids University of California

Santa Barbara, Santa Barbara, CA 93106, USA

E-mail: ajhe@physics.ucsb.edu

Dr. S. R. Cowan

National Renewable Energy Lab

1617 Cole Blvd., Golden, CO 80401, USA

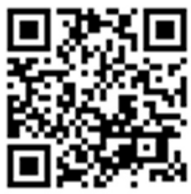

DOI: 10.1002/adfm.201101632 solutions to fit particular applications. For example, polymer photovoltaic technology is well-suited to provide power to residential and commercial structures via semitransparent windows or opaque roof coatings. As illustrated in Figure 1a, polymer solar cells can be produced in large areas on plastic substrates, demonstrating that manufactured modules can be flexible, light weight, robust, and ideal for a wide variety of un-tethered energy applications where portability and durability are key features.

Advances in optical and electrical performance and stability have been complemented by progress in the physical understanding of the device science of BHJ solar cells. ${ }^{[20]}$ Understanding the complex physical processes governing energy generation within organic BHJ solar cells will play an important role in materials and device optimization as the field continues to mature. ${ }^{[21-26]}$ Here, we aim to provide a critical discussion of the operation of $\mathrm{BHJ}$ solar cells based on recent experimental results, with focus on charge generation, collection, and recombination processes.

The BHJ material is composed of a "donor" organic material (semiconducting polymer or small molecule) that absorbs light efficiently and an "acceptor" electron-transporting material (fullerene, polymer, or small molecule) that facilitates charge transfer from the donor material. The donor and 


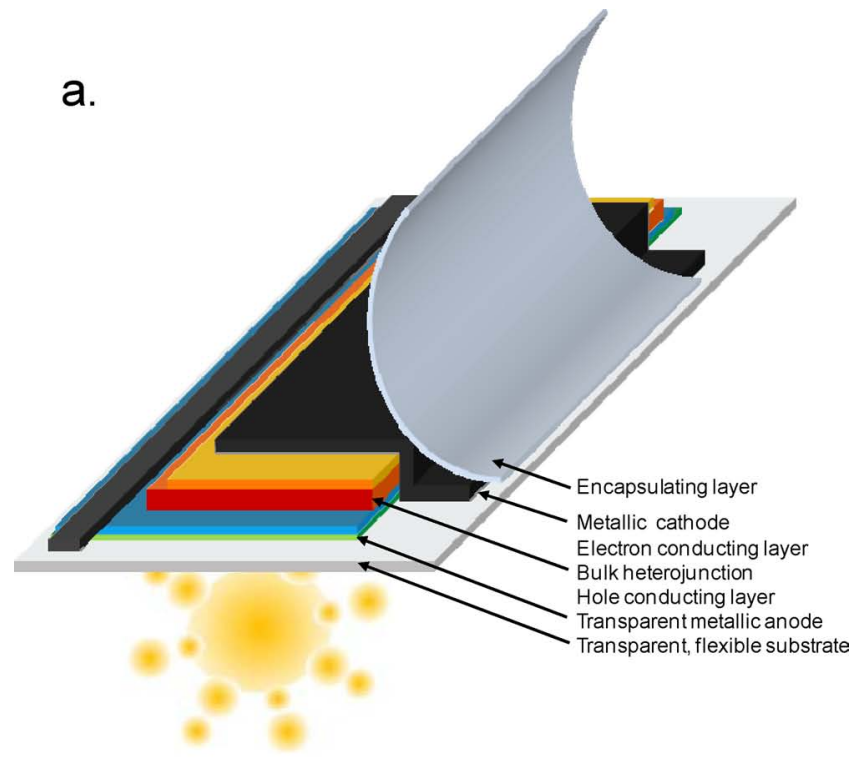

b.

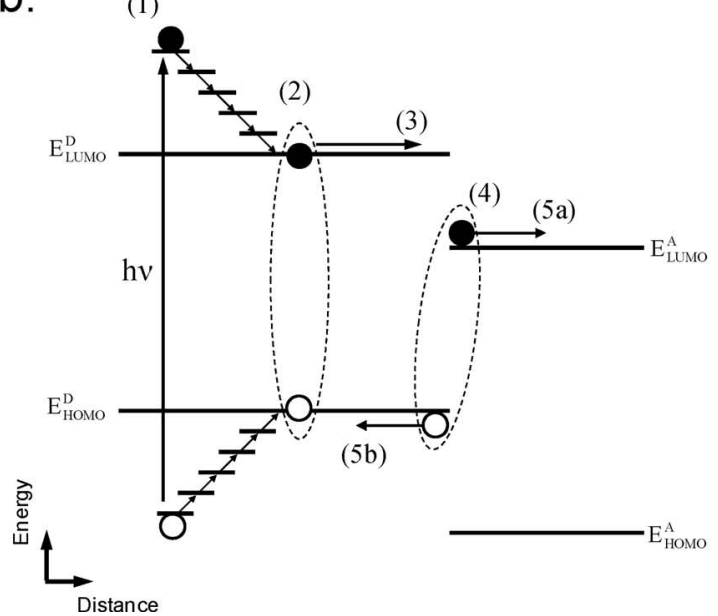

Figure 1. a) Device structure of a typical non-inverted organic $\mathrm{BH}$ J solar cell. b) Commonly accepted outline of the physical processes converting absorbed photons to collected current: the photoexcitation (1) thermalizes to the band edge to form a bound exciton (2); this exciton diffuses (3) to a $\mathrm{BH}$ ) interface and charge transfers (4); and mobile electrons (5a) and holes (5b) are transported to the anode and cathode, respectively. However, the time scale of these processes is not consistent: exciton diffusion is too slow to account for ultrafast charge transfer.

acceptor components are blended, with phase separation on the nanoscale (thought to be $10-20 \mathrm{~nm}$ in the most efficient systems ${ }^{[27-32]}$ ) to form donor and acceptor domains. The photogenerated charge carriers reach the electrodes by transport through the phase separated (but percolated) domains.

Photons incident upon a "plastic" solar cell initiate a series of optical and electrical processes ultimately resulting in the generation of charge in the external circuit. A photon absorbed by a polymer chain produces an excitation along the conjugated backbone. ${ }^{[20]}$ Debate about the nature of the primary photoexcitation is still on-going, ${ }^{[20,25,33-36]}$ and it has been repeatedly demonstrated that the excited state undergoes a series of relaxation

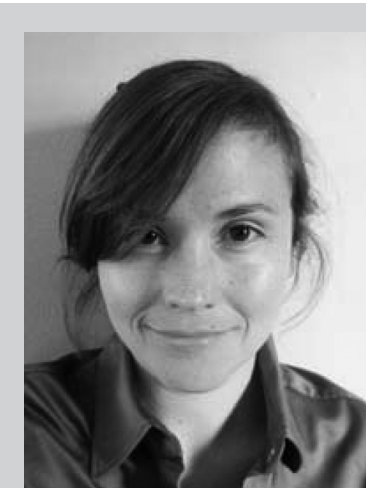

Sarah R. Cowan obtained her Ph.D. in materials science and engineering from the University of California, Santa Barbara, studying recombination mechanisms, device engineering, and device physics in organic solar cells with Professor Heeger. She is currently an EERE SunShot postdoctoral research fellow at the National Renewable Energy Laboratory in Golden, CO. Her research interests include designing functional contacts for organic multijunction device architectures.

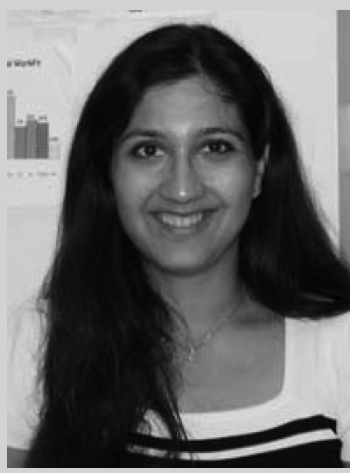

Natalie Banerji joined the group of Professor Heeger at the University of California, Santa Barbara, in 2009 as a postdoctoral research fellow. Prior to this, she obtained her Ph.D. in chemistry at the University of Geneva, Switzerland. Her research interests include ultrafast spectroscopy, photophysics of organic solar cell materials, and optoelectronic properties of conjugated polymers. She has very recently moved to the Ecole Polytechnique Fédérale de Lausanne (EPFL), Switzerland.

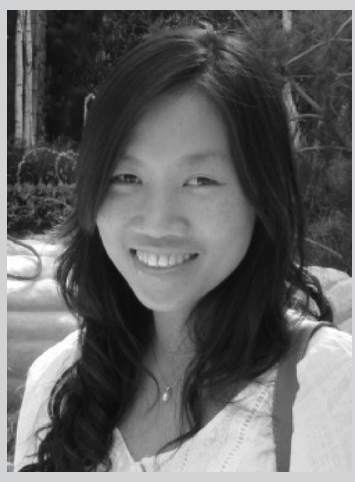

Wei Lin Leong received her Ph.D. degree in materials science and engineering from Nanyang Technological University, Singapore in 2010. In 2010, she joined Professor Heeger's group. Her research work comprises developing solution-processed solar cells based on polymers or small molecules as well as understanding charge loss mechanism and effect of trap states. Her other research interests include organic transistors and memories.

processes. ${ }^{[37-53]}$ Charge transfer at the donor/acceptor interface splits the excitation into a domain-segregated electron and hole. Many time-resolved studies have shown that the charge transfer to the fullerene materials is nearly quantitative and ultrafast (<100 fs for many optimized systems). ${ }^{[54-63]}$ Based on relaxation time scales found during time-resolved fluorescence 
studies on pristine polymers in our group, we have pointed out that the ultrafast charge separation can compete with relaxation processes such as excited-state localization and exciton diffusion. ${ }^{[37,38]}$ Moreover, charge transfer on the order of $100 \mathrm{fs}$ (or less) limits exciton diffusion to the $0.1-0.2 \mathrm{~nm}$ length scale, according to published exciton diffusion coefficients. ${ }^{[64,65]}$ However, nanoscale phase separation ${ }^{[27-32]}$ is known to be necessary for efficient bulk heterojunction photovoltaics, ${ }^{[66]}$ so that there is a discrepancy between the distance that an exciton can diffuse and the distance that it needs to diffuse in order to ensure ultrafast charge separation at the fullerene interface. The second section of this article will discuss an alternative mechanism in which the primary photoexcitation, which is distinctively different from the bound exciton since it is more delocalized and consists of a mobile electron and hole, reaches an interface and undergoes charge separation.

A large fraction of the incident light absorbed in the $\mathrm{BHJ}$ in optimized devices is converted to photoinduced charge carriers that are collected as current in an external circuit. The photogenerated current is reduced by internal recombination loss prior to charge collection at the electrodes. Competition between carrier sweep-out by the internal field and the loss of photogenerated carriers by recombination is one of the key issues to overcome for high efficiency devices. Due to the relatively low charge carrier mobility in common $\mathrm{BHJ}$ materials, the power conversion efficiency does not scale linearly with the thickness of the active layer in polymer BHJ solar cells. As the active layer thickness increases, the path length of photogenerated electrons and holes to the electrodes increases. Thin devices can be made with almost $100 \%$ conversion efficiency of absorbed photons to electrons collected in the external circuit. ${ }^{[1]}$ However, increasing the thickness of the active layer in these devices typically does not increase overall efficiency. ${ }^{[6,67]}$ The solar cell active layer thickness which maximizes power conversion efficiency is usually smaller than necessary for optimal light harvesting. The short circuit current increases moderately (non-linearly) with increasing active layer thicknesses, ${ }^{[68]}$ but often, the open circuit voltage is reduced and the fill factor drops dramatically. While a larger fraction of the incident photons are absorbed in thick-film devices, the resulting photogenerated charge carriers are not efficiently extracted into the external circuit.

Charge recombination becomes the dominant loss mechanism as the active layer thickness increases, limiting short circuit current, open circuit voltage, and fill factor. Unfortunately, as researchers seek to increase the efficiency of polymer photovoltaics and companies begin the process of upscaling this technology, increasing the thickness of the active layer of the $\mathrm{BHJ}$ becomes necessary due to the minimum film thicknesses that can be reproducibly fabricated from scalable printing processes (roll-to-roll printing, etc.). ${ }^{[6,69]}$

Charge recombination has been studied via a variety of experimental methods, including time-of-flight (TOF), ${ }^{[70]}$ steady-state current-voltage, ${ }^{[71-74]}$ impedance spectroscopy, ${ }^{[75-77]}$ photoinduced charge extraction by linearly increasing voltage (photo-CELIV), ${ }^{[78]}$ double injection currents, ${ }^{[79]}$ transient absorption, and transient photovoltage measurements. ${ }^{[80,81]}$ At applied voltages above the maximum power point and at the open circuit condition, bimolecular recombination reduces the current density and limits the fill factor, thereby decreasing power conversion efficiency. ${ }^{[1,81]}$ Determining primary recombination mechanisms and their physical origins are therefore essential to the future of organic BHJ solar cells. The third and fourth sections of this article summarize the results of recent experiments designed to probe charge carrier sweep-out and the nature of recombination in organic solar cells.

\section{Photoexcitation and Charge Transfer}

Figure $1 \mathrm{~b}$ visualizes the commonly used physical picture of current generation in $\mathrm{BHJ}$ solar cells. This includes: 1) absorption of a photon, 2) thermalization of the primary excitation and formation of a singlet exciton, 3) exciton diffusion to a donoracceptor interface, 4) charge transfer to create electrons and holes, and 5) charge transport to the electrodes. The exciton binding energy $\left(E_{\mathrm{b}}\right)$ in semiconducting polymers is intermediate between that of highly delocalized Wannier-Mott excitons in inorganic semiconductors (usually less than the thermal voltage, $V_{\mathrm{T}}=k T / q \approx 25 \mathrm{mV}$ at room temperature, due to electric field screening in the material ${ }^{[20]}$ ) and localized Frenkel excitons in molecular crystals. Contrasting values for $E_{\mathrm{b}}$ for conjugated polymers ranging from $0.05 \mathrm{eV}$ (in poly(phenylene vinylenes) $)^{[20]}$ to $\approx 0.4 \mathrm{meV}^{[82-84]}$ have been reported. According to the above-mentioned mechanism, diffusion and separation of tightly bound excitons limit the photoinduced charge transfer at the heterojunction interfaces between the donor and acceptor phases, which is necessary for efficient current generation.

Let us examine the mechanism depicted in Figure $1 \mathrm{~b}$ in more detail. Charge separation in the poly(2-methoxy-5- $\left(3^{\prime}-7^{\prime}\right.$ dimethyloctyloxy)-1,4-phenylenevinylene) (MDMO-PPV):[6,6]phenyl- $\mathrm{C}_{61}$-butyric acid methyl ester (PCBM) composite has been directly measured to occur within $45 \mathrm{fs}$ after photoexcitation. ${ }^{[57]}$ Some references exist that point to much slower charge separation, with a distribution of time constants that is correlated to the distribution of exciton distances from the interface. ${ }^{[85,86]}$ Nevertheless, most femtosecond transient absorption studies carried out on polymer:fullerene blends with optimized composition and morphology evidence the appearance of charges faster than the 100-200 fs time resolution of the experiments. ${ }^{[54-59,63,87]}$

Admitting that the charge separation indeed occurs on the $100 \mathrm{fs}$ time scale, this implies that exciton diffusion to a fullerene interface also occurs in $<100 \mathrm{fs}$. According to the exciton diffusion coefficients reported in literature for poly[2methoxy-5-(2'-ethyl-hexyloxy)-p-phenylene vinylene] (MEHPPV) and poly-3(hexylthiophene) (P3HT) $\left(3 \times 10^{-3} \mathrm{~cm}^{2} \mathrm{~s}^{-1}\right.$ and $1.8 \times 10^{-3} \mathrm{~cm}^{2} \mathrm{~s}^{-1}$, respectively), excitons can diffuse by only $0.1-0.2 \mathrm{~nm}$ in $100 \mathrm{fs}^{\left[{ }^{[64,65]}\right.}$ This is clearly much less than the typical length scale of about 10-20 nm in optimized bulk heterojunctions, estimated by structural characterization in optimized morphologies. ${ }^{[27-32]}$ Furthermore, point-like exciton diffusion by the hopping mechanism has been measured on numerous occasions to take $0.5-1$ ps for a single hopping step, ${ }^{[39-42,44-46,49-51,88,89]}$ which is again too slow to explain how excitons reach a fullerene interface in $<100 \mathrm{fs}$. In agreement with this, an ultrafast transient absorption study of poly[2,7-(9,9-dioctylfluorene)-alt-5,5-(4,7'-di-2-thienyl-2', $1^{\prime}, 3$ benzothiadiazole)] (APFO3) films with various concentrations 
of PCBM revealed that exciton hopping before charge separation only occurs in films with very low fullerene concentration and poor morphology, but does not play a role for favorable blends having ultrafast quantitative charge separation within 200 fs. $^{[87]}$

One could argue that the $10 \mathrm{~nm}$ length scale for phase separation in the $\mathrm{BHJ}$ is largely overestimated, and that the donor and acceptor are in reality so intimately mixed that every excitation in the polymer occurs so close to a fullerene that no diffusion is necessary prior to charge separation. Against this speaks to the experimental observation that power conversion efficiency in very intimately mixed polymer:fullerene blends is often low because charge transport is inefficient. For example for the polymer poly(2,5-bis(3-alkylthiophen-2-yl)thieno[3,2-b] thiophene) (PBTTT), the fullerene intercalates into the crystalline polymer phase, but very low power conversion efficiency (PCE) was observed with the intimately mixed blend in spite of complete fluorescence quenching. ${ }^{[0]}$ Good solar cell performance for this polymer could only be achieved with a high fullerene concentration leading to phase separation and pure fullerene domains for electron transport. Also, as-cast films of P3HT:PCBM have been shown to display intimate mixing and have a PCE of less than $1 \% .{ }^{[91]}$ This can be increased to $5 \%$ by thermal annealing, which increases the phase separation and leads to more crystalline polymer domains of about $10 \mathrm{~nm}$. A recent publication claims that the charge separation in annealed P3HT:PCBM blends is slower (around $3 \mathrm{ps)}$ due to the increased domain size compared to non-annealed films, where charges are formed within hundreds of femtoseconds. ${ }^{[92]}$ The authors nevertheless point out that even in the annealed case the charge separation rate is still orders of magnitude higher than if it was truly limited by point-like exciton migration. They conclude that excitons must be highly spatially delocalized in the ordered P3HT domains, so that they can sample distances of the order of $10 \mathrm{~nm}$ on a very fast time scale-see Figure 2a.

In our opinion, it is a crude oversimplification to assign the excited state of conjugated polymers to a unique species, such as an exciton, without considering its temporal evolution. We have shown in two recently published studies that complex relaxation processes occur in the excited state of conjugated polymers, spanning time scales from femtoseconds to hundreds of picoseconds. ${ }^{[37,38]}$ In BHJ blends, charge separation at the donor/acceptor interface and relaxation in the pure polymer phase are therefore competing processes. Banerji et. al. investigated pristine polymers poly[ $N$-9 $9^{\prime \prime}$-hepta-decanyl-2,7-carbazolealt-5,5-(4',7'-di-2-thienyl-2', $1^{\prime}, 3^{\prime}$-benzothiadiazole) (PCDTBT) and $\mathrm{P} 3 \mathrm{HT}$ by femtosecond-resolved fluorescence up-conversion spectroscopy. ${ }^{[37,38]}$ The results with both polymers point to the formation of an initially delocalized photoexcitation which localizes on the $100 \mathrm{fs}$ time scale and then relaxes to a bound state in approximately $1 \mathrm{ps}$. This is followed by successive exciton migration steps (hopping) to lower energy localized states, which exist as a result of disorder (0.5-100 ps). In parallel, there is also slow conformational relaxation of the polymer backbone (up to $\approx 200 \mathrm{ps}$ in thin films).

The initial localization of the primary photoexcitation, evidenced in the fluorescence up-conversion study by an ultrafast red-shift of the emission spectrum and loss of polarization anisotropy, has been independently reported by several groups,
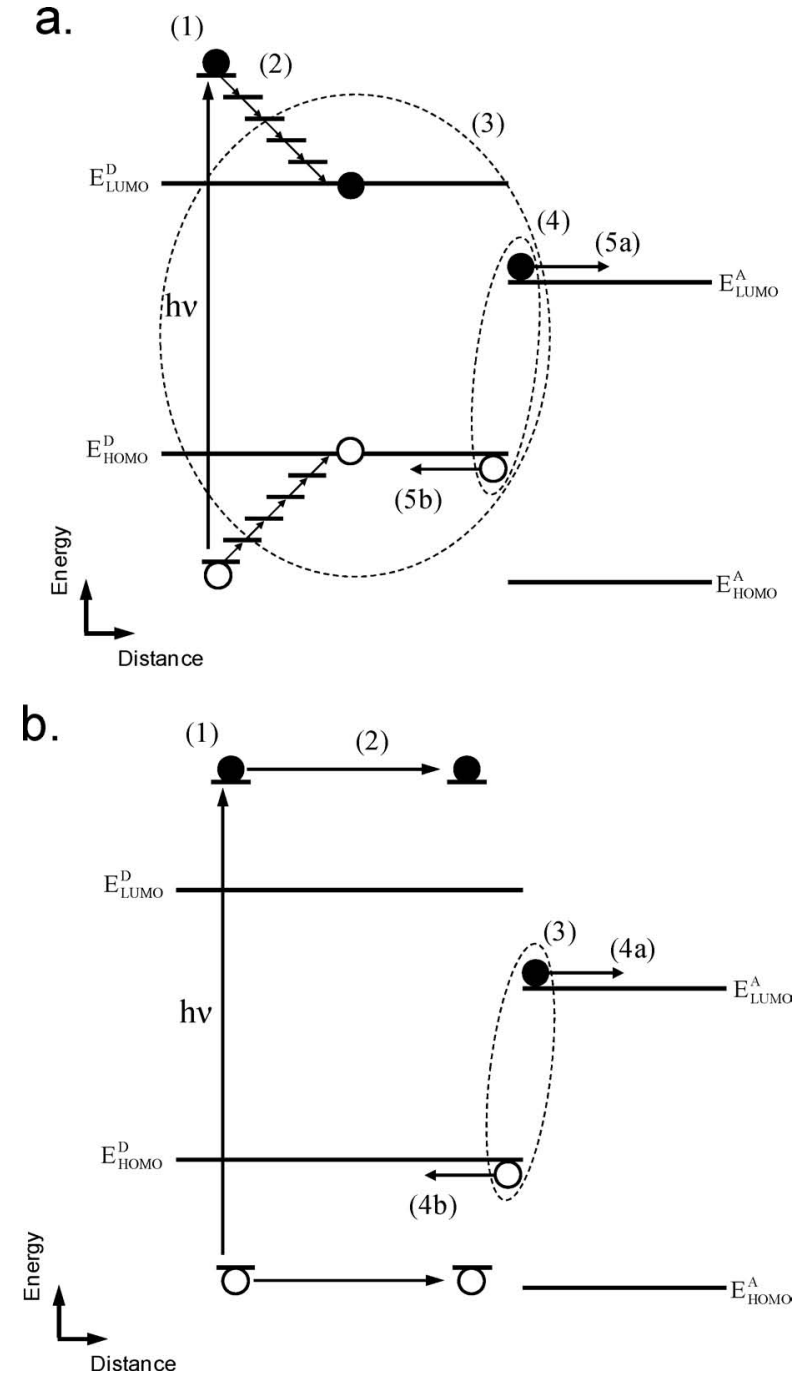

Figure 2. Two proposed mechanisms for ultrafast charge separation at a fullerene interface following polymer photoexcitation in organic $\mathrm{BH}$ ) solar cells: a) The primary photoexcitation (1) thermalizes to the band edges (2) to form a bound exciton; This delocalized singlet exciton (3) can sample spatial distances of $1 \mathrm{~nm}$ to $10 \mathrm{~nm}$, resulting in ultrafast charge transfer (4) and mobile carrier generation (5a,b). b) The highly delocalized primary photoexcitation containing mobile electrons and holes $(1,2)$ charge transfers (3) before thermalization and exciton formation in less than $100 \mathrm{fs}$ and mobile carriers $(4 \mathrm{a}, \mathrm{b})$ are formed in the two phases.

using for example photon-echo experiments. ${ }^{[39,40,43,47,48,53,93-95]}$ It is largely driven by local geometrical relaxation from the more twisted geometry of the ground state to a planar quinoidal structure in the excited state. ${ }^{[4,48,52,53,93-95]}$ A delocalization of the primary photoexcitation over at least $11 \mathrm{~nm}$ has been reported for MEH-PPV. ${ }^{[48]}$ Although the primary photoexcitation in conjugated polymers is often referred to as an exciton ${ }^{[33]}$ or hot exciton, ${ }^{[96]}$ we do not believe that the binding of the opposite charges in this non-thermalized and highly delocalized initial state is strong enough to formally call it an exciton. Instead, we use the semiconductor formalism and describe it as a correlated, but not bound, pair of a mobile electron and hole, formed by a $\pi-\pi *$ interband transition. This semiconductor picture is 
also supported by previous experimental results obtained in our group from highly oriented MEH-PPV chains in a polyethylene matrix, ${ }^{[97-100]}$ and on evidence obtained from photoconductivity studies and ultrafast investigations of the infrared active vibrational (IRAV) modes in poly(p-phenylene vinylene) (PPV) and MEH-PPV. ${ }^{[101-106]}$ The thermalized, more localized and Coulomb bound exciton state is only formed later, following relaxation, on a time scale that we estimate to $1 \mathrm{ps}$ based on an early rise of the emission spectrum in the fluorescence up-conversion data. ${ }^{[37,38]}$

We mentioned before the discrepancy between the distance that a polymer exciton can diffuse in $100 \mathrm{fs}(0.1-0.2 \mathrm{~nm})$ and the distance that the photoexcitation needs to cover $(\approx 10 \mathrm{~nm})$ in order to reach a fullerene interface in the $\mathrm{BHJ}$ to undergo ultrafast ( $<100 \mathrm{fs}$ ) charge separation. Based on a comparison between the time scales for the various relaxation processes occurring in the excited polymer and the time scale of charge separation, we infer that the $<100 \mathrm{fs}$ charge transfer to the fullerene occurs before exciton formation, hopping and conformational relaxation, in fact during the localization of the primary photoexcited species. We therefore propose an alternative mechanism that is consistent with ultrafast charge separation during intraband relaxation in the polymer, before localization of the primary excitation to form a bound exciton, as outlined in Figure $2 \mathrm{~b}$. It seems very plausible that the electrons and holes formed in the conduction and valence band are more delocalized and/or mobile directly after the interband absorption than the relaxed excitons that are both spatially localized (by local structural relaxation of the polymer) and bound by the Coulomb interaction into an exciton state. In the proposed picture, the primary excitation can therefore reach the interface faster than a relaxed exciton, which could account for the ultrafast charge separation in BHJ blends. Furthermore, the exciton binding energy does not have to be overcome because there is excess energy in the system at the time of charge separation.

Assuming, according to our picture, initially delocalized electrons and holes, and transport to the interface (before self-localization) by drift in a field of order $10^{6} \mathrm{~V} \mathrm{~cm}^{-1}$, an electron mobility of approximately $10 \mathrm{~cm}^{2} \mathrm{~V}^{-1} \mathrm{~s}^{-1}$ would be required for carriers to move $10 \mathrm{~nm}$ in $100 \mathrm{fs}$ (see Figure 2b). Let us examine whether such a high transient mobility in the ultrafast time regime is reasonable. Indeed, timeresolved terahertz spectroscopy revealed initial carrier mobilities in P3HT film up to 30-40 $\mathrm{cm}^{2} \mathrm{~V}^{-1} \mathrm{~s}^{-1}$.107] Moreover, transient photoconductivity measurements in polymer: fullerene blends have demonstrated a continuing decrease of carrier mobility after photoexcitation even at significantly longer time scales. ${ }^{[08]}$ The mobility of non-relaxed carriers directly after photoexcitation should therefore not be compared to steady-state mobility of structurally relaxed polarons (typically, $<<1 \mathrm{~cm}^{2} \mathrm{~V}^{-1} \mathrm{~s}^{-1}$ for conjugated polymers in BHJ phase separated materials). In addition, wave function delocalization over several repeat units will assist the transport of the carriers to the interface. Quantum mechanical transport of delocalized primary excitations has been demonstrated by Collini and Scholes by the experimental observation of coherent excitation energy migration within MEH-PPV chains in solution, with transport over distances sufficient to reduce polarization memory in the 50-100 fs time regime. ${ }^{[109]}$

We conclude that diffusion of a bound and relaxed exciton is unlikely to be involved in the charge transfer mechanism in polymer:fullerene BHJ blends, because it is too slow. The much debated concepts of exciton diffusion length and exciton binding energy therefore may be less important for charge separation in "plastic" solar cells than has been commonly assumed. Carrier delocalization before exciton formation with high initial mobilities and transport assisted by quantum effects offer a plausible explanation to the observation that provides the foundation for the entire field of BHJ solar cells: ultrafast photoinduced charge transfer.

\section{Competition Between Carrier Sweep-Out and Recombination: Role of the Internal Field in Charge Collection}

In a bulk heterojunction photodiode under illumination, excess charges are created in the polymer and fullerene domains due to photoexcitation and subsequent charge transfer, as discussed in the previous section. Mobile (free) carriers must then be generated and collected at opposite electrodes prior to recombination, as temporally described in Figure 3, in order to contribute to measured current. Recombination lifetimes of the charge carriers in the donor and acceptor domains limit the efficient extraction of these carriers to an external circuit. While recombination can be minimized in optimized bulk heterojunction thin films (BHJ thickness $<100 \mathrm{~nm}$ ), ${ }^{[1]}$ recombination increases as the BHJ film thickness is increased, ${ }^{[6,67]}$ likely due to the low carrier mobility in the disordered donor and acceptor materials.

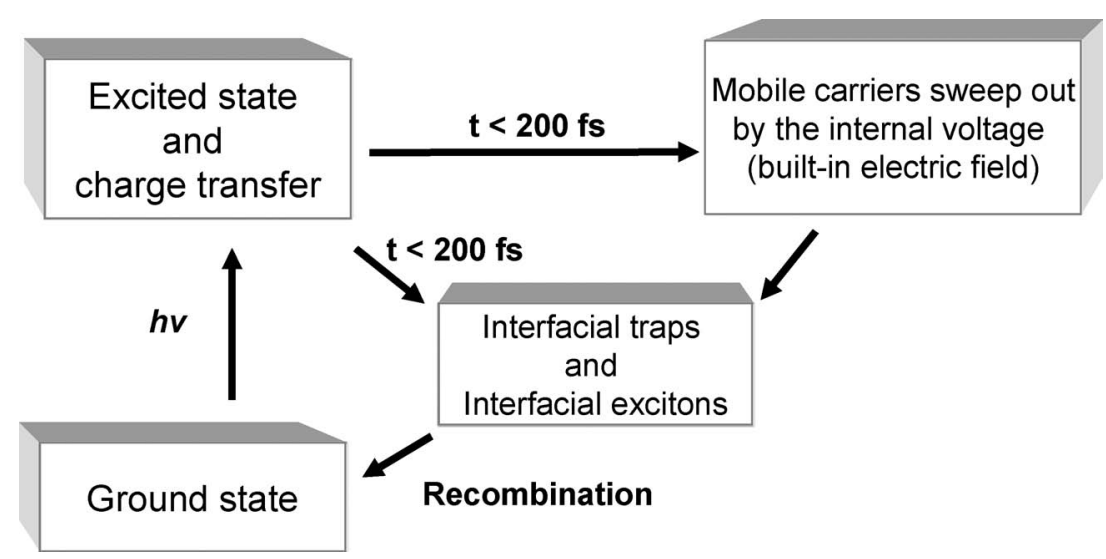

Figure 3. Time dynamics during the operation of the $\mathrm{BHJ}$ solar cell. Photoexcitation is followed by ultrafast charge transfer that creates mobile carriers in the donor and acceptor domains. These carriers are subsequently either swept out to the electrodes by the built-in electric field or they recombine to the ground state. The sweep-out time is less than $1 \mu \mathrm{s}$ at short circuit where the recombination probability is small. For efficient solar cells, the recombination time must be long compared to the sweep-out time. Reproduced with permission. ${ }^{[14]}$ Copyright 2011, American Physical Society. 
The strong internal field present within BHJ solar cells at short circuit conditions has been stipulated to affect both the generation of free charge carriers, ${ }^{[63,110-112]}$ their loss due to various recombination processes ${ }^{[81,113]}$ and "sweep-out"-the process by which charge carriers escape recombination to be collected at the electrodes. ${ }^{[114-116]}$ Results from transient absorption spectroscopy have often been interpreted according to a model in which charge transfer from the polymer to the fullerene leads to the formation of a Coulomb bound interfacial charge pair (also called interfacial exciton, polaron pair, ${ }^{[117]}$ or intermediate charge transfer state, Figure 3). ${ }^{[11,118-120]}$ The charge pair either undergoes geminate recombination or dissociates into free charge carriers that can ultimately be collected at the electrodes. The presence of an internal electric field at the short circuit condition has long been considered necessary for the separation of the strongly bound charge pair. Although this seems to be confirmed by the observed electric field-dependence of free charge generation, ${ }^{[112]}$ some ultrafast transient absorption experiments have shown the generation of free carriers on the order to picoseconds, too fast to be interpreted as field-dependent charge separation. ${ }^{[121,122]}$ As the meaningful interpretation of transient absorption results is made difficult by high-intensity excitation conditions, broad/ overlapping spectral signatures, and complexity in assigning the bands, alternative techniques have been applied. Kneipert and colleagues use a new experimental method to study photocurrent extraction called time-delayed collection field (TDCF). ${ }^{[15]}$ TDCF measurements resolve photocurrent extraction on the nanosecond to microsecond timescale using an optical excitation pulse and time-delayed electric field extraction. Generated charge density is found to be independent of the applied electric field during illumination, indicating that charge separation is not a field-dependent process. Similarly, more investigations will be discussed in Section 4, which show that recombination in BHJ solar cells is bimolecular or trap-based, rather than geminate. Therefore, the possibility that charge transfer from the polymer to the fullerene directly yields free charge carriers (without an intermediate bound state that leads to geminate recombination) should be seriously considered.

While the role of the internal field in free charge generation is questionable, it certainly influences the collection efficiency of charges at the electrodes. Recent experimental results suggest that under high electric field conditions (short circuit current, $J_{\mathrm{sc}}$, and reverse bias), mobile carriers are efficiently swept out of the $\mathrm{BHJ}$ via drift transport on the order of $\approx 100 \mathrm{~ns}$. ${ }^{[114,115]}$ This is again consistent with evidence of fast generation of free charge carriers ${ }^{[37,111,122]}$ because efficient charge collection must occur before recombination can significantly reduce the free charge carrier population. Results from a variety of transient photocurrent techniques on functional polymer solar cells are consistent with carrier transport at a constant drift velocity at short circuit. ${ }^{[14,115,123]}$ Here, we explore evidence for the idea that efficient charge collection at the short circuit condition and in reverse bias in polymer solar cells is due to free carriers transported via drift in a strong built-in electric field, a process known in photodiodes as sweep-out, ${ }^{[114,124]}$ and the role of the internal field in charge collection at the short circuit condition. The sweep-out competes efficiently with non-geminate recombination so that charges can be collected.

While sweep-out is a well-known phenomenon in photodiodes, it has only recently been shown to play a large role in efficient charge collection in polymer solar cells in reverse bias and at the short circuit condition where the internal electric field can be high. Charge sweep-out is a classical concept and when applied to BHJ materials requires optimized charge extraction characteristics within the $\mathrm{BHJ}$ for charge to drift. Inefficient BHJ morphologies or inefficient charge collection at the contacts can lead to highly non-uniform spatially distributed charge densities, sometimes called space charge, where the electric field is locally screened and diffusion may play a larger role in transport. In disordered films, regions of localized charge are likely sites for increased recombination. Within a classical picture of charge transport, charge carriers with average carrier mobility, $\mu$, drifting in an electric field, $E$, will transit a given distance, $d$, in a characteristic time, $\tau_{\mathrm{sw}}$, the sweep-out time. Here, we imagine a carrier traversing the $\mathrm{BHJ}$ and define $d$ equal to the BHJ thickness. The drift velocity, $v$, of these carriers is classically given as $v=\mu E=d \tau_{\mathrm{sw}}$. The electric field across a given distance can be estimated as the internal voltage, $V_{\text {int }}$, per unit length, so long as the internal voltage is uniform across the distance. While photoconductive atomic force microscopy (AFM) measurements reveal morphologydependent nanoscale variations in the electric field, ${ }^{[125]}$ we assume that these variations produce a nearly uniform average field on the macroscale. Hence, the sweep-out time is defined as $\tau_{\text {sw }}=d^{2} / 2 \mu V_{\text {int }}$, inversely proportional to the internal voltage. A geometric factor (here, 2) is inserted to account for uniform charge generation across the $\mathrm{BHJ}$ layer. Charge carriers must travel on average through only one half the length of the BHJ to be collected.

Transient photocurrent experiments by Cowan et al. show this to be a good model of charge extraction over a wide range of internal voltages in $\mathrm{BHJ}$ solar cells composed of PCDTBT:PC ${ }_{71} B M$ ([6,6]-phenyl- $C_{71}$-butyric acid methyl ester) and P3HT:PCBM. ${ }^{[114]}$ Experiments were performed on solar cell architectures by pulsing a laser with $10 \mathrm{~ns}$ pulse duration and $1 \mathrm{~Hz}$ repetition rate. Current was measured by the voltage drop across a small resistor in series with the cell. Representative bias-dependent data is plotted in the inset of Figure 4, where $V$ is the externally applied voltage. Internal voltage is determined by the equation $V_{\text {int }}=V_{\mathrm{BI}}-V$, where $V_{\mathrm{BI}}$ is the built-in voltage and sometimes called the flatband voltage elsewhere. $V_{\mathrm{BI}}$ is estimated from the voltage at which the transient photocurrent as a function of voltage changes sign. Carrier decay times are extracted from the transient photocurrent decay data and are shown in Figure 4 for the two $\mathrm{BHJ}$ systems and two temperatures studied. The sweep-out time is found to be proportional to the inverse internal voltage from reverse bias (low inverse internal voltages) to approaching the maximum power point. At short circuit and room temperature, charge is extracted from both BHJ systems in $<1 \mu \mathrm{s}$. Transient photoconductance data in Figure 4 imply the existence of a well-defined macroscopic internal field; carrier sweep-out is proportional to the magnitude of this internal field and is limited by the carrier mobility. Other experiments have 


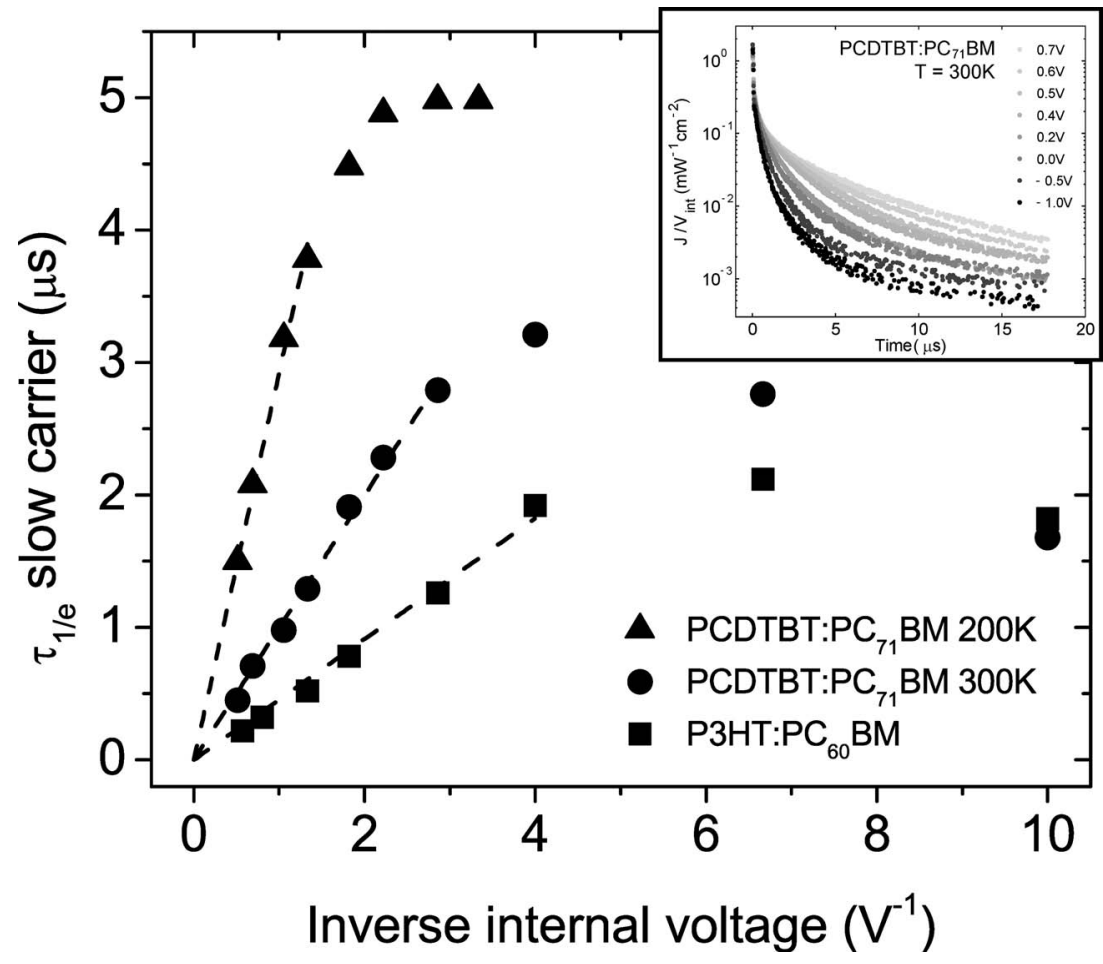

Figure 4. Estimated transit time of slow carriers vs. the inverse internal voltage $\left(1 / V_{\text {int }}\right)$. In the sweep-out limit (high internal voltage), data are fit to the drift mobility equation, $\tau_{\mathrm{sw}}=d^{2} / 2 \mu V_{\text {int }}$. Inset: transient photoconductance vs. time for the PCDTBT: $\mathrm{PC}_{71} \mathrm{BM}$ cell (temperature, $T=300 \mathrm{~K}$ ). Voltage applied to the cell is scanned from $-1.0 \mathrm{~V}$ to $0.7 \mathrm{~V}$. Reproduced with permission. ${ }^{[114]}$ Copyright 2011, American Physical Society.

qualitatively observed drift transport under high field conditions, observed as linear charge extraction. ${ }^{[115,123]}$ At external voltages near open circuit where the internal field approaches zero, the transit time can no longer be described solely by drift transport- non-geminate recombination plays a large role, and recombination is bias-dependent.

Our conclusions concerning the bias dependence of nongeminate charge recombination were recently confirmed by Mauer et al., who developed a novel experimental technique, a time-delayed dual pulse (TDDP) current-voltage measurement, which directly probed the dwell time of charge carriers in P3HT:PCBM devices. ${ }^{[16]}$ Quasi-steady-state currentvoltage curves were recorded as a function of time delay, intensity, and bias upon irradiation with two pulsed lasers, and the results were extrapolated to solar illumination. Nongeminate recombination, identified specifically as bimolecular recombination in many recent reports, is measured to be bias dependent. Low recombination is found at the short circuit condition, in agreement with high current collection in the extraction regime. Current loss due to bimolecular recombination increases with applied bias, and at the low internal fields present at the open circuit condition, a large fraction of charge bimolecularly recombines. These new results and experimental method will be discussed in more detail in Section 4.

At the short circuit condition, Mauer et al. report very small bimolecular recombination loss under 1 sun illumination conditions, ${ }^{[116]}$ a result in agreement with differential current measurements performed by Koster and colleagues this year. ${ }^{[76]}$ Relatively small current loss due to bimolecular recombination (2\% to $17 \%$ depending on sample preparation in these experiments) at the short circuit condition has thus far been overlooked ${ }^{[72,126]}$ because of efficient carrier collection in this regime. In the efficient charge collection regime, $J_{\mathrm{sc}}$ is thought to be dominated by charge carrier sweep-out via drift transport in the high internal electric field, ${ }^{[114]}$ as described by the expression for drift current, $J_{n_{\mathrm{e}}}=q \mu n_{\mathrm{e}} E$, where $q$ is the elementary unit of charge, $n_{\mathrm{e}}$ is the density of electrons (or holes assuming $n_{\mathrm{e}} \approx n_{\mathrm{h}}$ ), and $E$ is the internal electric field. The expression for drift current predicts that short circuit current is linearly proportional to the density of photogenerated carriers, which has been shown to increase proportional to incident light intensity. ${ }^{[113]}$ Due to high levels of charge collection, small amounts $(<10 \%)$ of bimolecular current loss cause only small deviations from the intensity-dependent linearity of $J_{\mathrm{sc}}$. Hence, the dependence of steadystate short circuit current on incident light intensity, $I, J_{\mathrm{sc}} \propto I^{\alpha}$, where the physical interpretation of the fitting parameter, $\alpha$, is under debate, is not a high-precision measure of recombination mechanism as sometimes thought. ${ }^{[127]}$ In the common interpretation of the fitting parameter, $\alpha$, fit values of $\alpha=1$ are attributed to first order loss processes, and the values of $\alpha=0.5$ to second order (bimolecular) loss processes. ${ }^{[127]}$ In recent literature, space charge effects were found to predict $\alpha=0.75 .{ }^{[126]}$ Koster and colleagues improve upon these models and present an analytically derived expression for the fraction of carriers lost to bimolecular recombination, $\eta_{\mathrm{BR}}$, in relation to the steady-state fitting parameter, $\alpha: \eta_{\mathrm{BR}}=\alpha^{-1}-1$. It is worthwhile noting that when $\alpha=0.5$, the current, $J_{\mathrm{sc}}$, is reduced by one order of magnitude, rather than completely recombining. However, it is still true that in this regime, current loss is dominated by bimolecular recombination. This new expression for bimolecular recombination loss efficiency, as well as differential current density measurements, should prove to be a very useful tool in the determination of current loss in the extraction regime.

We conclude that there is interesting recent evidence to suggest the presence of a uniform electric field drop across the BHJ layer, as a result of the built-in electric field and applied bias voltage. High charge carrier collection at the short circuit condition and in reverse bias results from drift transport of photogenerated charge within this uniform electric field. Carrier extraction may be described by drift transport of charge to the contacts over a wide range of relevant applied bias voltages. Additionally, bimolecular (non-geminate) recombination can account for current loss at the short circuit condition. Finally, bimolecular recombination is found to be bias dependent, a concept we will explore in further detail in the next section. 


\section{Bias- and Charge-Density-Dependent Charge Carrier Recombination: Bimolecular Recombination Takes the Foreground}

Although research into the role of exciton recombination and geminate recombination via Coulomb bound interfacial charge pairs (see Section 3) is ongoing, ${ }^{[111,112,121,122,128]}$ an interesting new hypothesis has emerged: that bimolecular recombination accounts for the majority of recombination from short circuit to open circuit, ${ }^{[76,115,116]}$ and that bimolecular recombination is bias and charge-density dependent. ${ }^{[72,81,115,116]}$ This new hypothesis is notably interesting within the context of previous work, because it presupposes highly efficient charge formation and separation, ${ }^{[128]}$ and that recombination through traps, either from material impurities ${ }^{[71,74]}$ or unoptimized morphology, ${ }^{[129]}$ does not appear to be a major loss mechanism in optimized cells.

Recombination of photogenerated charge carriers in polymer BHJ solar cells reduces the short circuit current, the fill factor and the open-circuit voltage. ${ }^{[72]}$ Identifying the mechanisms of recombination is, therefore, fundamentally important for increasing the power conversion efficiency. Bimolecular recombination, the interdomain band-to-band transition, as sketched in Figure 5a, involves two mobile and independent charge carriers, and hence the recombination rate, $R$, can be considered a second order process with respect to the mobile charge carrier density $\left(R \propto n_{\mathrm{e}}{ }^{2}\right)$. In a device with trap-free electron and hole transport and a high density of photogenerated charge carriers, mobile carriers are most likely to recombine non-radiatively via bimolecular recombination. Trap-assisted recombination can be characterized by recombination of mobile charge with a trapped carrier (see Figure $5 \mathrm{~b}$ ) and hence can be considered a first order process with respect to the density of mobile charge $\left(R \propto n_{\mathrm{h}}\right.$, in the case of an electron trap). The presence of impurities and defects that function as traps enhances trap-assisted recombination, as will be described below. In disordered donor:acceptor blends, ${ }^{[130,131]}$ it may be necessary to go beyond the classical trap-assisted Shockley-Read-Hall recombination theory; for example SRH recombination may occur through a distribution of states in the disordered band tails and through trap states with energy levels influenced by their local environment in the BHJ matrix as shown in Figure 5c.

Light-intensity-dependent current-voltage measurements on polymer $\mathrm{BHJ}$ cells made from a variety of different semiconducting polymers and fullerenes show that the kinetics are voltage dependent ${ }^{[72,81,115-116]}$ and evolve from charge carrier sweep-out in the extraction regime (reverse bias and short circuit) to a carrier loss regime around open circuit. Bimolecular recombination dominates the charge kinetics as a result of increasing, bias-dependent charge carrier density in the cell. ${ }^{[72,81]}$ Bimolecular recombination is almost universally identified as the dominant recombination mechanism in optimized organic bulk heterojunction solar cells at the open circuit condition. ${ }^{[72,73,81,132]}$ However, due to an overwhelming background of charge carrier sweep-out, a study of recombination at the short circuit condition has been experimentally challenging until recently, as discussed in the previous section. Two new experimental methods have been implemented to study recombination across the whole current-voltage $(J-V)$ curve and have

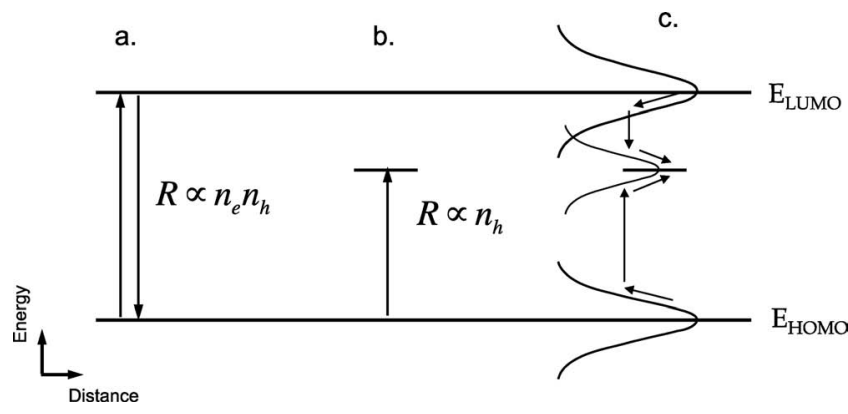

Figure 5. Recombination mechanisms of excess carriers: a) Bimolecular (band-to-band) recombination; b) Trap-assisted recombination visualized as an electron trap. A density of free holes, $n_{\mathrm{h}}$, recombine with a population of trapped electron charge, $n_{\mathrm{e}, \mathrm{t}}$; and c) $\mathrm{SRH}$ recombination through bandtail states and trap states influenced by the local environment in disordered materials.

identified bimolecular recombination as the dominant current loss mechanism across the pertinent range of bias voltages.

Using time-delayed collection field experiments, ${ }^{[115]}$ Kneipert et al. probe charge collection and recombination dynamics via a "pump-probe" analogue: a diode-pumped, Q-switched neodymium-doped yttrium aluminium garnet (Nd:YAG) laser with 8 ns pulse width generates charge within a P3HT:PCBM BHJ solar cell under a bias voltage. After a time delay, allowing for charge to dynamically interact within the device, a high extraction bias $(V=-5 \mathrm{~V})$ is applied, pulling charge out of the device. Integrated photocurrent transients recorded upon extraction with variable time delay, $t_{\mathrm{d}}$, from $t_{\mathrm{d}}=100 \mathrm{~ns}$ to $10 \mu \mathrm{s}$ allow analysis of the evolution of charge extraction and recombination within the device. There are two important observations to make from the data with respect to bimolecular recombination. First, at the short circuit condition, the applied pre-bias voltage provides enough extraction field to efficiently extract most of the excited charge population. Given a delay time of $10 \mu \mathrm{s}$ (the longest delay time probed), $\approx 15 \%$ of the initially excited charge recombines before collection. As pre-bias is increased, the total charge population decreases as delay time is increased. This prebias and delay time dependent reduction in charge density is well-reproduced by incorporating Langevin recombination ${ }^{[70,133]}$ as the loss mechanism. Bimolecular (Langevin-type) recombination increases from $\approx 17 \%$ at short circuit to $\approx 58 \%$ at a prebias of $0.55 \mathrm{~V}$, just slightly above the open circuit condition.

Mauer and colleagues came to similar conclusions using another unique experimental method: time-delayed dual pulse (TDDP) current-voltage measurements. ${ }^{[116]}$ TDDP probes P3HT:PCBM BHJ solar cell devices using two pulsed lasers with total fluence energy comparable to solar illumination. By varying the time delay between the two pulsed lasers, the interaction time of two charge populations is controlled. When the delay between pulses is long, two separate charge populations transit the $\mathrm{BHJ}$ layer, resulting in an equivalent collected current equal to twice the current collected from one pulse. A delay time of $9 \mu \mathrm{s}$ is necessary to collect the total current, or in other words, most charge is extracted after excitation (100 fs pulse length is reported) in $\approx 10 \mu \mathrm{s}$. As the time delay between pulses is decreased, the complementary charge populations are both present in the $\mathrm{BHJ}$ layer simultaneously. In the case of 
low delay times, these interacting charge populations recombine at higher rate than the individual charge populations. Additionally, applied bias is again seen to reduce the dwell time of charge when reverse bias is applied. As bias increases toward open circuit, charge is extracted more slowly, which is in agreement with results from Cowan et al.[114] Longer dwell times, in addition to increased charge density, result in increased current loss. The measured charge-density- and bias-dependent recombination rates are again well-reproduced via a Langevin-type bimolecular recombination model. Representative data in Figure $\mathbf{6}$ show bias-dependent bimolecular recombination under steady-state conditions. ${ }^{[116]}$ Bimolecular recombination increases with decreasing internal field, and this experiment implies that the increased recombination may be due to increased dwell times and higher charge densities. Additionally, in concert with recent results from other groups, these results indicate that the effect of bimolecular recombination should not be neglected at either the short circuit condition or at open circuit.

We finish this article by further discussing recent evidence for charge density dependence of bimolecular recombination. To the authors' knowledge, few reports have been published to date concerning the charge density dependence of bimolecular recombination. Bimolecular recombination will occur more frequently where populations of electrons and holes intersect, as the energetic transition occurs without mediation through a localized state, which could increase the probability of opposite charge species becoming correlated. Charge extraction and transient photovoltage experiments by C. G. Shuttle, et al. have offered the most direct look into the $\mathrm{BHJ}$ charge density. ${ }^{[80,81,113,134]}$ Additionally, varying the intensity of light incident on a $\mathrm{BHJ}$ solar cell has long been assumed to linearly increase the charge density. ${ }^{[113]}$ In a paper published last year, C. G. Shuttle and colleagues used charge extraction techniques to probe the bias dependence of the BHJ charge density, as shown in Figure 7. ${ }^{[13]}$ Increasing charge density proportional to applied bias can be attributed to two effects: a) increasing externally applied bias decreases the internal field for charge extraction, localizing photogenerated charge carriers and b) the potential barrier for charge injection from the contacts decreases via the applied voltage. Beyond the diode's turn-on voltage, a significant density of charge is injected from the contacts. Charge extraction experiments measure both mobile and trapped charge populations in the active layer, but due to the transient nature of the experiments, only observe effects from the photogenerated current.

One may estimate the difference in charge density between short circuit and open circuit in a relatively optimized device with dynamics dominated by drift current sweep-out at short circuit and bimolecular recombination at open circuit. ${ }^{[72]}$ At short circuit, the photocurrent, $J_{\mathrm{sc}}=J_{\text {photo }}=q d G P_{\mathrm{C}}(V=0)$, where $q$ is the elementary unit of charge, $d$ is the thickness of the bulk heterojunction, $G$ is the photon flux absorbed by the solar cell per unit volume, and $P_{\mathrm{C}}$ is the charge collection probability. $P_{\mathrm{C}}$ approaches 1 at short circuit in the most efficient systems. If the current at short circuit is determined by drift, then $J_{\mathrm{sc}}=2 q \mu n_{\mathrm{sc}} E=q d G$, where $n_{\mathrm{sc}}$ is the electron (or hole) charge density at short circuit. This equation can be rewritten

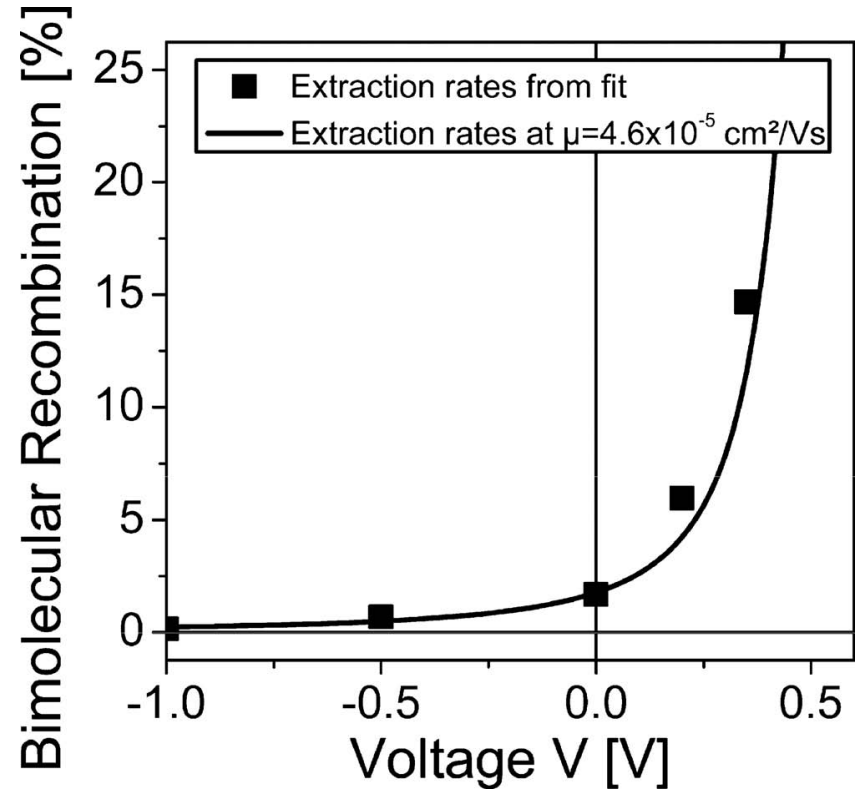

Figure 6. The bimolecular recombination rate, as determined from timedelayed dual pulse experiments, is found to increase non-linearly with increasing bias in a $\mathrm{P} 3 \mathrm{HT}$ :PCBM BHJ solar cell. Increasing recombination rate data (black squares) can be reproduced using the drift-diffusion model incorporating Langevin recombination (black line), and using an average mobility as seen in the graph. Reproduced with permission. [116] Copyright 2011, American Chemical Society.

in terms of the time scale for sweep-out, $\tau_{\mathrm{sw}}: G=n_{\mathrm{sc}} / \tau_{\mathrm{sw}}$. If we assume that all the photogenerated charge recombines bimolecularly at open circuit, $G=R\left(V_{\mathrm{oc}}\right)=\gamma m_{\mathrm{oc}}$, then we can relate charge density at open circuit, $n_{\mathrm{oc}}$, to charge density at short circuit, $n_{\mathrm{sc}}: n_{\mathrm{sc}} / \tau_{\mathrm{sw}}=m_{\mathrm{oc}}{ }^{2}$. Using $n_{\mathrm{oc}} / n_{\mathrm{sc}}=25$ from Figure 7

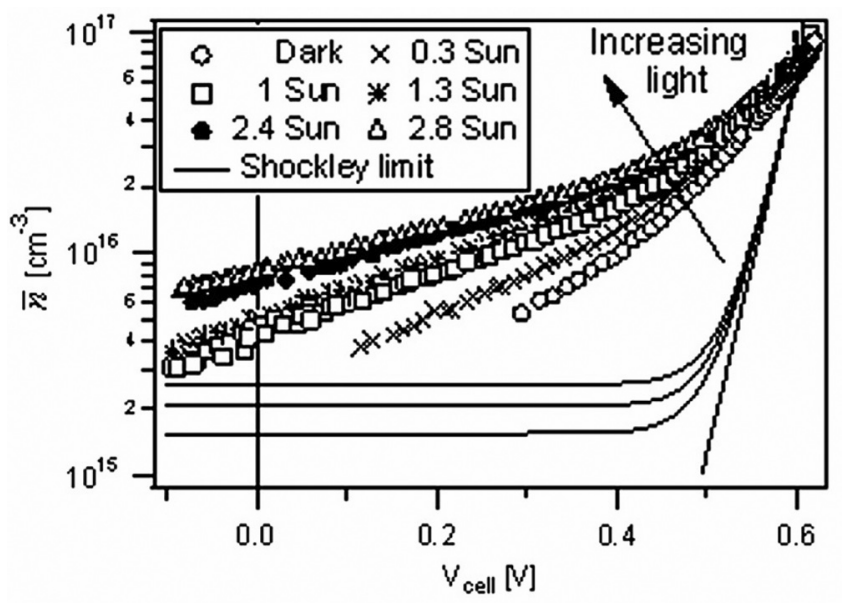

Figure 7. Bias dependence of the charge density of a P3HT:PCBM BHJ solar cell is plotted with varying incident light intensity. Charge density, as determined from charge extraction experiments, is found to increase more than an order of magnitude from short circuit to open circuit. Measured charge density dependence varies significantly from the Shockley limit (black lines). Reproduced with permission. [113] Copyright 2010, National Academy of Sciences, USA. 
for a P3HT:PCBM BHJ, ${ }^{[113]}$ and $\tau_{\mathrm{sw}} \approx 1 \mu \mathrm{s}^{[114]}$ for a similarly prepared P3HT:PCBM BHJ solar cell, the bimolecular recombination coefficient $\gamma$ can be calculated entirely from data to be $\gamma=4 \times 10^{-13} \mathrm{~cm}^{3} \mathrm{~s}^{-1}$. The bimolecular recombination coefficient has been reported to be in the range from $10^{-12} \mathrm{~cm}^{3} \mathrm{~s}^{-1}>$ $\gamma>10^{-14} \mathrm{~cm}^{3} \mathrm{~s}^{-1}$ implying that the ratio of charge density at open circuit to short circuit may be in the range $10>n_{\mathrm{oc}} / n_{\mathrm{sc}}>$ 1000 for polymer solar cells constructed of other materials or via other fabrication processes. Increasing charge densities can be correlated with increased bimolecular recombination, as indicated by TDDP current-voltage measurements, although further experiments will be necessary to determine the relationship between charge density and recombination rate and strength.

One such study published this year investigates the charge-density and trap-density dependence of the recombination mechanism in polymer BHJ solar cells. ${ }^{[71]}$ We note that while bimolecular recombination has been identified as the dominant recombination mechanism in optimized $\mathrm{BHJ}$ solar cells, investigations into the recombination mechanisms of unoptimized devices (both in material purity and morphology) will remain relevant as long as the optimization of devices using newly synthesized materials continues to play a role in forward progress of the field. In order to probe the recombination mechanism, small concentrations of impurity were introduced into PCDTBT:PCBM BHJ solar cells. It was found that impurity levels of even 1 part in 1000 altered the electronic properties of the $\mathrm{BHJ}$ devices, reducing the open circuit voltage, the short circuit current, and the fill factor. Steady-state current-voltage studies showed a dramatic increase in the trap-assisted recombination rate when $\mathrm{PC}_{84} \mathrm{BM}\left([6,6]\right.$-phenyl- $\mathrm{C}_{84}$-butyric acid methyl ester) was introduced as a trap site into the polymer BHJ solar cells. ${ }^{[71]}$ This trap density-dependent recombination was modeled as a sum of bimolecular and Shockley-Read-Hall recombination processes; the latter was dramatically enhanced by the addition of the $\mathrm{PC}_{84} \mathrm{BM}$ traps. Results of trapping and recombination discussed here are confirmed in the source literature, ${ }^{[71,77]}$ via transient photocurrent and transient absorption and impedance spectroscopy.

The trap-assisted recombination rate (proportional to the trap density) and the generation rate (proportional to incident light intensity) control the cross-over from bimolecular to trap-assisted recombination, as plotted (both data and model) in Figure 8 . Cross-sections of Figure 8 at a single trap density reproduce the commonly plotted intensity dependence of the open circuit voltage. ${ }^{[72,73]}$ Above the cross-over point at low trap densities and high light intensities, the open circuit voltage is relatively unaffected by the density of traps within the $\mathrm{BHJ}$. Below the cross-over point at high trap densities, such as those potentially found in BHJ materials with impurities left over from the synthesis and device processing, recombination through traps becomes the dominant loss mechanism and results in a significant reduction in the open circuit voltage at operating illumination levels.

The dominant recombination mechanism is both incident light intensity dependent (proportional to charge density) and trap density dependent (inversely proportional to the free carrier lifetime). Here, we offer an interpretation of this data.

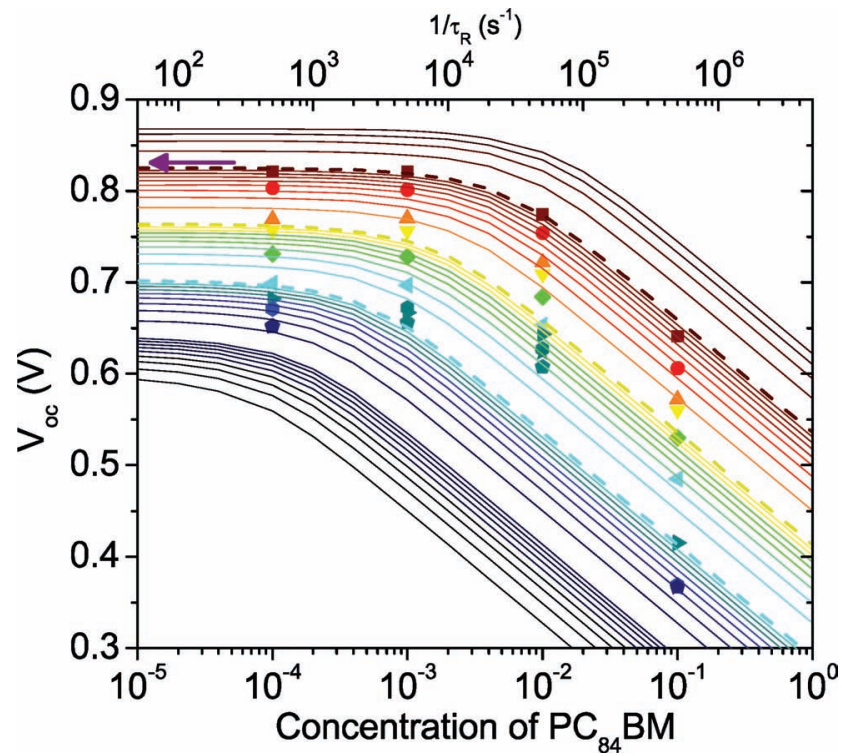

Figure 8. Intensity/charge-density dependent $V_{\text {oc }}$ plotted as a function of $\mathrm{PC}_{84} \mathrm{BM}$ trap concentration (data points, lower $x$-axis) in a PCDTBT:PCBM $\mathrm{BHJ}$ solar cell. Bimolecular/SRH recombination model fits to the data (lines, upper $x$-axis): the trap-assisted recombination rate plotted vs. the generation rate of photoexcitations with color from blue to brown indicating an increase in generation rate. Arrow indicates the $V_{o c}$ at 1 sun for the trap-free PCDTBT:PCBM solar cell devices. Dotted lines correspond to logarithmic increases in generation rate $\left(G\right.$ (brown) $=10^{21} \mathrm{~cm}^{-3} \mathrm{~s}^{-1}$, $G$ (yellow) $=10^{20} \mathrm{~cm}^{-3} \mathrm{~s}^{-1}, G$ (blue) $=10^{19} \mathrm{~cm}^{-3} \mathrm{~s}^{-1}$ ). Reproduced with permission. ${ }^{[71]}$

At high incident light intensity, the higher density of charge carriers increases the probability of bimolecular recombination (an electron and a hole must find each other in the $\mathrm{BHJ}$ material). At low light intensity, charge carriers are typically farther apart and traps are more likely to be empty, making them viable routes for recombination. Increasing the trap density increases the probability of trap-assisted recombination. In practice, trap states are likely to result from impurities, polymerization defects, and poor phase separation (unoptimized formation of the BHJ material). Further research into the physical mechanisms of charge recombination may not only lead to more highly efficient organic BHJ solar cells, but may also decrease the time necessary to evaluate newly synthesized materials.

\section{Outlook}

We have presented a critical discussion of the charge photoexcitation and excited-state relaxation in PCDTBT and P3HT polymers and discussed a fresh picture of the charge transfer mechanism (before relaxation) in BHJ blends. Next, the role of internal field in the $\mathrm{BHJ}$ in charge collection of photogenerated carriers and the interplay between charge sweep-out and recombination were discussed. Finally, we explored recent emphasis on bimolecular recombination in the literature, and the bias- and charge-density- and trap density-dependence of the recombination mechanism. Organic solar cells will likely 
exceed power conversion efficiencies over $10 \%$ in the near future as predicted by theoretical analysis ${ }^{[35]}$ and demonstrated with monochromatic incident light. ${ }^{[1]}$ Efficient photoinduced charge transfer and minimal recombination are required to produce efficient polymer BHJ solar cells. We hope this discussion of charge transfer, recombination, and collection dynamics will lead to deeper fundamental understanding of the device science and, hopefully, be a step toward establishing the criteria for achieving the "ideal" solar cell.

\section{Acknowledgements}

This research was supported by the US Army General Technical Services and the Department of Energy (DOE FG02-08ER46535). S.C. was supported by the Center for Energy Efficient Materials, an Energy Frontier Research Center funded by the U.S. Department of Energy, Office of Science, Office of Basic Energy Sciences under award Number DE-SC0001009. N.B. thanks the Swiss National Science Foundation for Fellowship support (fellowship for prospective researchers PBGEP2125859). W.L.L. is grateful to the Agency for Science, Technology and Research ( $A *$ Star) of Singapore for a postdoctoral fellowship.

Received: July 15, 2011 Revised: October 15, 2011 Published online: January 31, 2012

[1] S. H. Park, A. Roy, S. Beaupre, S. Cho, N. Coates, J. S. Moon, D. Moses, M. Leclerc, K. Lee, A. J. Heeger, Nat. Photonics 2009, 3, 297.

[2] Konarka Technologies Inc., Konarka's Power Plastic Achieves World Record 8.3\% Efficiency Certification from National Energy Renewable Laboratory (NREL), Nov. 29, 2010. http://www.konarka. com/index.php/site/pressreleasedetail/konarkas_power_plastic_ achieves_world_record_83_efficiency_certification_fr.

[3] BusinessWire, Solarmer Energy, Inc. Breaks Psychological Barrier with $8.13 \%$ OPV Efficiency, July 27, 2010, http://www.businesswire. $\mathrm{com} /$ news/home/20100727005484/en/Solarmer-Energy-BreaksPsychological-Barrier-8.13-OPV.

[4] Y. Liang, Z. Xu, J. Xia, S.-T. Tsai, Y. Wu, G. Li, C. Ray, L. Yu, Adv. Mater. 2010, 22, E135.

[5] N. Blouin, A. Michaud, D. Gendron, S. Wakim, E. Blair, R. Neagu-Plesu, M. Belletete, G. Durocher, Y. Tao, M. Leclerc, J. Am. Chem. Soc. 2008, 130, 732.

[6] Y. Sun, C. J. Takacs, S. R. Cowan, J. H. Seo, X. Gong, A. Roy, A. J. Heeger, Adv. Mater. 2011, 23, 2226.

[7] E. Voroshazi, B. Verreet, T. Aernouts, P. Heremans, Sol. Energy Mater. Sol. Cells 2011, 95, 1303.

[8] M. S. White, D. C. Olson, S. E. Shaheen, N. Kopidakis, D. S. Ginley, Appl. Phys. Lett. 2006, 89, 143517.

[9] J. A. Hauch, P. Schilinsky, S. A. Choulis, R. Childers, M. Biele, C. J. Brabec, Sol. Energy Mater. Sol. Cells 2008, 92, 727.

[10] F. C. Krebs, Sol. Energy Mater. Sol. Cells 2009, 93, 394.

[11] C. N. Hoth, S. A. Choulis, P. Schilinsky, C. J. Brabec, Adv. Mater. 2007, 19, 3973.

[12] F. C. Krebs, M. Jorgensen, K. Norrman, O. Hagemann, J. Alstrup, T. D. Nielsen, J. Fyenbo, K. Larsen, J. Kristensen, Sol. Energy Mater. Sol. Cells 2009, 93, 422

[13] S. E. Shaheen, R. Radspinner, N. Peyghambarian, G. E. Jabbour, Appl. Phys. Lett. 2001, 79, 2996.

[14] C. Girotto, B. P. Rand, J. Genoe, P. Heremans, Sol. Energy Mater. Sol. Cells 2009, 93, 454
[15] K. X. Steirer, M. O. Reese, B. L. Rupert, N. Kopidakis, D. C. Olson, R. T. Collins, D. S. Ginley, Sol. Energy Mater. Sol. Cells 2009, 93, 447

[16] F. C. Krebs, Sol. Energy Mater. Sol. Cells 2009, 93, 465.

[17] S. S. Kim, S. I. Na, J. Jo, G. Tae, D. Y. Kim, Adv. Mater. 2007, 19, 4410.

[18] K. Norrman, A. Ghanbari-Siahkali, N. B. Larsen, Annu. Rep. Prog. Chem. Sect. C: Phys. Chem. 2005, 101, 174.

[19] P. Schilinsky, C. Waldauf, C. J. Brabec, Adv. Funct. Mater. 2006, 16, 1669.

[20] A. J. Heeger, N. S. Sariciftci, E. B. Namdas, Semiconducting and Metallic Polymers, Oxford University Press, Oxford, UK 2010.

[21] C. J. Brabec, S. Gowrisanker, J. J. M. Halls, D. Laird, S. Jia, S. P. Williams, Adv. Mater. 2010, 22, 3839.

[22] C. Deibel, V. Dyakonov, Rep. Prog. Phys. 2010, 73, 096401.

[23] C. Deibel, V. Dyakonov, C. J. Brabec, IEEE J. Sel. Top. Quantum. Electron 2010, 16, 1517.

[24] G. Dennler, M. C. Scharber, C. J. Brabec, Adv. Mater. 2009, 21, 1323.

[25] A. J. Heeger, Chem. Soc. Rev. 2010, 39, 2354.

[26] M. Helgesen, R. Sondergaard, F. C. Krebs, J. Mater. Chem. 2010, 20, 36 .

[27] D. Chen, A. Nakahara, D. Wei, D. Nordlund, T. P. Russell, Nano Lett. 2010, 11, 561.

[28] M. Dante, A. Garcia, T. Q. Nguyen, J. Phys. Chem. C 2009, 113, 1596.

[29] T. Erb, U. Zhokhavets, G. Gobsch, S. Raleva, B. Stuhn, P. Schilinsky, C. Waldauf, C. J. Brabec, Adv. Funct. Mater. 2005, 15, 1193.

[30] J. S. Moon, C. J. Takacs, S. Cho, R. C. Coffin, H. Kim, G. C. Bazan, A. J. Heeger, Nano Lett. 2010, 10, 4005.

[31] A. J. Parnell, A. J. Cadby, O. O. Mykhaylyk, A. D. F. Dunbar, P. E. Hopkinson, A. M. Donald, R. A. L. Jones, Macromolecules 2011, 44, 6503

[32] B. Walker, A. B. Tomayo, X. D. Dang, P. Zalar, J. H. Seo, A. Garcia, M. Tantiwiwat, T. Q. Nguyen, Adv. Funct. Mater. 2009, 19, 3063.

[33] I. G. Scheblykin, A. Yartsev, T. Pullerits, V. Gulbinas, V. Sundstrom, J. Phys. Chem. B 2007, 111, 6303.

[34] W. Barford, Electronic and optical properties of conjugated polymers, Oxford University Press, Oxford, UK 2005.

[35] G. Lanzani, G. Cerullo, D. Polli, A. Gambetta, M. Zavelani-Rossi, C. Gadermaier, Phys. Status Solidi A 2004, 201, 1116.

[36] N. S. Sariciftci, Primary photoexcitations in conjugated polymers: Molecular exciton versus semiconductor band model, World Scientific, Singapore 1997.

[37] N. Banerji, S. Cowan, M. Leclerc, E. Vauthey, A. J. Heeger, J. Am. Chem. Soc. 2010, 132, 17459.

[38] N. Banerji, S. Cowan, E. Vauthey, A. J. Heeger, J. Phys. Chem. C 2011, 115, 9726

[39] M. M. L. Grage, T. Pullerits, A. Ruseckas, M. Theander, O. Inganas, V. Sundstrom, Chem. Phys. Lett. 2001, 339, 96.

[40] M. M. L. Grage, Y. Zaushitsyn, A. Yartsev, M. Chachisvilis, V. Sundstrom, T. Pullerits, Phys. Rev. B 2003, 67, 205207.

[41] G. R. Hayes, I. D. W. Samuel, R. T. Phillips, Phys. Rev. B 1995, 52, 11569.

[42] G. R. Hayes, I. D. W. Samuel, R. T. Phillips, Synth. Met. 1997, 84, 889.

[43] T. E. Dykstra, V. Kovalevskij, X. J. Yang, G. D. Scholes, Chem. Phys. 2005, 318, 21.

[44] S. P. Kennedy, N. Garro, R. T. Phillips, Phys. Rev. B 2001, 6411, 115206.

[45] R. Kersting, U. Lemmer, R. F. Mahrt, K. Leo, H. Kurz, H. Bassler, E. O. Gobel, Phys. Rev. Lett. 1993, 70, 3820.

[46] R. Kersting, B. Mollay, M. Rusch, J. Wenisch, G. Leising, H. F. Kauffmann, J. Chem. Phys. 1997, 106, 2850. 
[47] F. Milota, J. Sperling, A. Tortschanoff, V. Szocs, L. Kuna, H. F. Kauffmann, J. Lumin. 2004, 108, 205.

[48] A. Ruseckas, P. Wood, I. D. W. Samuel, G. R. Webster, W. J. Mitchell, P. L. Burn, V. Sundstrom, Phys. Rev. B 2005, 72, 115214.

[49] J. Sperling, F. Milota, H. F. Kauffmann, Opt. Spectrosc. 2005, 98, 729.

[50] J. Sperling, F. Milota, A. Tortschanoff, C. Warmuth, B. Mollay, H. Bassler, H. F. Kauffmann, J. Chem. Phys. 2002, 117, 10877.

[51] C. Warmuth, A. Tortschanoff, K. Brunner, B. Mollay, H. F. Kauffmann, J. Lumin. 1998, 76-7, 498.

[52] N. P. Wells, B. W. Boudouris, M. A. Hillmyer, D. A. Blank, J. Phys. Chem. C 2007, 111, 15404.

[53] X. J. Yang, T. E. Dykstra, G. D. Scholes, Phys. Rev. B 2005, 71, 045203.

[54] K. G. Jespersen, F. L. Zhang, A. Gadisa, V. Sundstrom, A. Yartsev, O. Inganas, Org. Electron. 2006, 7, 235

[55] F. L. Zhang, K. G. Jespersen, C. Bjorstrom, M. Svensson, M. R. Andersson, V. Sundstrom, K. Magnusson, E. Moons, A. Yartsev, O. Inganas, Adv. Funct. Mater. 2006, 16, 667.

[56] A. A. Bakulin, J. C. Hummelen, M. S. Pshenichnikov, P. H. M. van Loosdrecht, Adv. Funct. Mater. 2010, 20, 1653.

[57] C. J. Brabec, G. Zerza, G. Cerullo, S. De Silvestri, S. Luzzati, J. C. Hummelen, S. Sariciftci, Chem. Phys. Lett. 2001, 340, 232.

[58] I. W. Hwang, J. Y. Kim, S. Cho, J. Yuen, N. Coates, K. Lee, M. Heeney, I. McCulloch, D. Moses, A. J. Heeger, J. Phys. Chem. C 2008, 112, 7853.

[59] I. W. Hwang, C. Soci, D. Moses, Z. G. Zhu, D. Waller, R. Gaudiana, C. J. Brabec, A. J. Heeger, Adv. Mater. 2007, 19, 2307.

[60] B. Kraabel, J. C. Hummelen, D. Vacar, D. Moses, N. S. Sariciftci, A. J. Heeger, F. Wudl, J. Chem. Phys. 1996, 104, 4267.

[61] S. Morita, A. A. Zakhidov, K. Yoshino, Solid State Commun. 1992, 82, 249.

[62] N. S. Sariciftci, L. Smilowitz, A. J. Heeger, F. Wudl, Science 1992, 258, 1474.

[63] M. Tong, N. E. Coates, D. Moses, A. J. Heeger, S. Beaupre, M. Leclerc, Phys. Rev. B 2010, 81, 125210.

[64] P. E. Shaw, A. Ruseckas, I. D. W. Samuel, Adv. Mater. 2008, 20, 3516

[65] A. J. Lewis, A. Ruseckas, O. P. M. Gaudin, G. R. Webster, P. L. Burn, I. D. W. Samuel, Org. Electron. 2006, 7, 452.

[66] M. Campoy-Quiles, T. Ferenczi, T. Agostinelli, P. G. Etchegoin, Y. Kim, T. D. Anthopoulos, P. N. Stavrinou, D. D. C. Bradley, J. Nelson, Nat. Mater. 2008, 7, 158.

[67] M. Lenes, Appl. Phys. Lett. 2006, 88, 243502.

[68] A. J. Moulé, K. Meerholz, Appl. Phys. B: Lasers Opt. 2007, 86, 721.

[69] J. Peet, L. Wen, P. Byrne, S. Rodman, K. Forberich, Y. Shao, N. Drolet, R. Gaudiana, G. Dennler, D. Waller, Appl. Phys. Lett. 2011, 98, 043301.

[70] A. Pivrikas, G. Juska, A. J. Mozer, M. Scharber, K. Arlauskas, N. S. Sariciftci, H. Stubb, R. Osterbacka, Phys. Rev. Lett. 2005, 94, 176806.

[71] S. R. Cowan, W. L. Leong, N. Banerji, G. Dennler, A. J. Heeger, Adv. Funct. Mater. 2011, 21, 3083.

[72] S. R. Cowan, A. Roy, A. J. Heeger, Phys. Rev. B 2010, 82, 245207.

[73] L. J. A. Koster, V. D. Mihailetchi, R. Ramaker, P. W. M. Blom, Appl. Phys. Lett. 2005, 86, 123509.

[74] M. M. Mandoc, F. B. Kooistra, J. C. Hummelen, B. de Boer, P. W. M. Blom, Appl. Phys. Lett. 2007, 91, 263505.

[75] G. Garcia-Belmonte, J. Bisquert, Appl. Phys. Lett. 2010, 96, 113301.

[76] L. J. A. Koster, M. Kemerink, M. M. Wienk, K. Maturová, R. A. J. Janssen, Adv. Mater. 2011, 23, 1670.

[77] W. L. Leong, S. R. Cowan, A. J. Heeger, Adv. Energy Mater. 2011, 1, 517.

[78] A. J. Mozer, G. Dennler, N. S. Sariciftci, M. Westerling, A. Pivrikas, R. Osterbacka, G. Juska, Phys. Rev. B 2005, 72, 035217.
[79] G. Juška, K. Arlauskas, J. Stuchlik, R. Österbacka, J. Non-Cryst. Solids 2006, 352, 1167.

[80] C. G. Shuttle, B. O'Regan, A. M. Ballantyne, J. Nelson, D. D. C. Bradley, J. de Mello, J. R. Durrant, Appl. Phys. Lett. 2008, 92, 093311.

[81] C. G. Shuttle, B. O'Regan, A. M. Ballantyne, J. Nelson, D. D. C. Bradley, J. R. Durrant, Phys. Rev. B 2008, 78, 113201.

[82] S. F. Alvarado, P. F. Seidler, D. G. Lidzey, D. D. C. Bradley, Phys. Rev. Lett. 1998, 81, 1082.

[83] E. M. Conwell, H. A. Mizes, Phys. Rev. B 1995, 51, 6953.

[84] C. Deibel, D. Mack, J. Gorenflot, A. Schöll, S. Krause, F. Reinert, D. Rauh, V. Dyakonov, Phys. Rev. B 2010, 81, 085202.

[85] L. W. Barbour, R. D. Pensack, M. Hegadorn, S. Arzhantsev, J. B. Asbury, J. Phys. Chem. C 2008, 112, 3926.

[86] L. W. Barbour, R. D. Pensack, M. Hegadorn, S. Arzhantsev, J. B. Asbury, J. Phys. Chem. C 2008, 112, 3926.

[87] S. De, T. Kesti, M. Maiti, F. Zhang, O. Inganaes, A. Yartsev, T. Pascher, V. Sundstroem, Chem. Phys. Lett. 2008, 350, 14.

[88] S. C. J. Meskers, J. Hubner, M. Oestreich, H. Bassler, J. Phys. Chem. B 2001, 105, 9139 .

[89] J. Pina, J. S. de Melo, H. D. Burrows, T. W. Bunnagel, D. Dolfen, C. J. Kudla, U. Scherf, J. Phys. Chem. B 2009, 113, 15928.

[90] N. C. Cates, R. Gysel, Z. Beiley, C. E. Miller, M. F. Toney, M. Heeney, I. McCulloch, M. D. McGehee, Nano Lett. 2009, 9, 4153.

[91] W. Ma, C. Yang, X. Gong, K. Lee, A. J. Heeger, Adv. Funct. Mater. 2005, 15, 1617.

[92] R. A. Marsh, J. M. Hodgkiss, S. Albert-Seifried, R. H. Friend, Nano Lett. 2010, 10, 923.

[93] Y. H. Lee, A. Yabushita, C. S. Hsu, S. H. Yang, I. Iwakura, C. W. Luo, K. H. Wu, T. Kobayashi, Chem. Phys. Lett. 2010, 498, 71

[94] J. Du, Z. Wang, W. Feng, K. Yoshino, T. Kobayashi, Phys. Rev. B 2008, 77, 195205.

[95] N. P. Wells, D. A. Blank, Phys. Rev. Lett. 2008, 100, 086403.

[96] V. I. Arkhipov, E. V. Emelianova, H. Bässler, Phy. Rev. Lett. 1999, 82, 1321.

[97] T. W. Hagler, K. Pakbaz, A. J. Heeger, Phys. Rev. B 1994, 49, 10968.

[98] T. W. Hagler, K. Pakbaz, A. J. Heeger, Phys. Rev. B 1995, 51, 14199.

[99] T. W. Hagler, K. Pakbaz, J. Moulton, F. Wudl, P. Smith, A. J. Heeger, Polym. Commun. 1991, 32, 339.

[100] T. W. Hagler, K. Pakbaz, K. F. Voss, A. J. Heeger, Phys. Rev. B 1991, 44, 8652

[101] P. B. Miranda, D. Moses, A. J. Heeger, Synth. Met. 2001, 119, 619.

[102] P. B. Miranda, D. Moses, A. J. Heeger, Phys. Rev. B 2001, 6408 $081201 / 1$

[103] D. Moses, A. Dogariu, A. J. Heeger, Phys. Rev. B 2000, 61, 9373.

[104] D. Moses, R. Schmechel, A. J. Heeger, Synth. Met. 2003, 139, 807.

[105] D. Moses, C. Soci, P. Miranda, A. J. Heeger, Chem. Phys. Lett. 2001, 350, 531.

[106] D. Moses, J. Wang, A. J. Heeger, N. Kirova, S. Brazovski, Proc. Natl. Acad. Sci. USA 2001, 98, 13496

[107] P. D. Cunningham, L. M. Hayden, J. Phys. Chem. C 2008, 112, 7928.

[108] N. E. Coates, D. Moses, A. J. Heeger, Appl. Phys. Lett. 2011, 98, 102103.

[109] E. Collini, G. D. Scholes, Science 2009, 323, 369

[110] I.-W. Hwang, D. Moses, A. J. Heeger, J. Phys. Chem. C 2008, 112, 4350.

[111] P. E. Keivanidis, T. M. Clarke, S. Lilliu, T. Agostinelli, J. E. Macdonald, J. R. Durrant, D. D. C. Bradley, J. Nelson, J. Phys. Chem. Lett. 2010 1, 734 .

[112] R. A. Marsh, J. M. Hodgkiss, R. H. Friend, Adv. Mater. 2010, 22, 3672.

[113] C. G. Shuttle, R. Hamilton, B. C. O'Regan, J. Nelson, J. R. Durrant, Proc. Natl. Acad. Sci. USA 2010, 107, 16448. 
[114] S. R. Cowan, R. A. Street, S. Cho, A. J. Heeger, Phys. Rev. B 2011, 83, 035205

[115] J. Kniepert, M. Schubert, J. C. Blakesley, D. Neher, J. Phys. Chem. Lett. 2011, 2, 700.

[116] R. Mauer, I. A. Howard, F. d. r. Laquai, J. Phys. Chem. Lett. 2011, 2, 1736.

[117] C. Deibel, T. Strobel, V. Dyakonov, Phys. Rev. Lett. 2009, 103, 036402.

[118] M. H. Tong, N. E. Coates, D. Moses, A. J. Heeger, S. Beaupre, M. Leclerc, Phys. Rev. B 2010, 81, 125210/1.

[119] I. W. Hwang, D. Moses, A. J. Heeger, J. Phys. Chem. C 2008, 112, 4350.

[120] S. De, T. Pascher, M. Maiti, K. G. Jespersen, T. Kesti, F. Zhang, O. Inganaes, A. Yartsev, V. Sundstroem, J. Am. Chem. Soc. 2007, 129, 8466.

[121] I. A. Howard, R. Mauer, M. Meister, F. d. r. Laquai, J. Am. Chem. Soc. 2010, 132, 14866

[122] J. Guo, H. Ohkita, H. Benten, S. Ito, J. Am. Chem. Soc. 2010, 132, 6154.

[123] N. S. Christ, S. W. Kettlitz, S. Valouch, S. Zufle, C. Gartner, M. Punke, U. Lemmer, J. Appl. Phys. 2009, 105.
[124] D. Moses, J. Wang, G. Yu, A. J. Heeger, Phys. Rev. Lett. 1998, 80, 2685.

[125] O. G. Reid, J. Appl. Phys. 2010, 108, 084320.

[126] L. J. A. Koster, V. D. Mihailetchi, H. Xie, P. W. M. Blom, Appl. Phys. Lett. 2005, 87.

[127] A. Rose, Concepts in Photoconductivity and Allied Problems, Wiley, New York 1963.

[128] F. Etzold, I. A. Howard, R. Mauer, M. Meister, T.-D. Kim, K.-S. Lee, N. S. Baek, F. d. r. Laquai, J. Am. Chem. Soc. 2011, 133, 9469.

[129] M. M. Mandoc, W. Veurman, L. J. A. Koster, B. de Boer, P. W. M. Blom, Adv. Funct. Mater. 2007, 17, 2167.

[130] J. Bisquert, G. Garcia-Belmonte, J. Phys. Chem. Lett. 2011, 2, 1950.

[131] R. A. Street, Phys. Rev. B 2011, 84.

[132] D. Credgington, R. Hamilton, P. Atienzar, J. Nelson, J. R. Durrant, Adv. Funct. Mater. 2011, 21, 2744.

[133] M. P. Langevin, Ann. Chim. Phys. 1903, 28, 433.

[134] C. G. Shuttle, A. Maurano, R. Hamilton, B. O'Regan, J. C. de Mello, J. R. Durrant, Appl. Phys. Lett. 2008, 93.

[135] T. Kirchartz, K. Taretto, U. Rau, J. Phys. Chem. C 2009, 113, 17958. 